\title{
DETECTING THE RISE AND FALL OF THE FIRST STARS BY THEIR IMPACT ON COSMIC REIONIZATION
}

\author{
Kyunguin Ahn ${ }^{1}$, Ilian T. Iliev ${ }^{2}$, Paul R. Shapiro ${ }^{3}$, Garrelt Mellema $^{4}$, Jun Koda ${ }^{5}$, and Yi Mao ${ }^{3}$ \\ ${ }^{1}$ Department of Earth Sciences, Chosun University, Gwangju 501-759, Republic of Korea; kjahn@ chosun.ac.kr \\ ${ }^{2}$ Astronomy Centre, Department of Physics \& Astronomy, Pevensey II Building, University of Sussex, Falmer, Brighton BN1 9QH, UK \\ ${ }^{3}$ Department of Astronomy and Texas Cosmology Center, University of Texas, Austin, TX 78712-1083, USA \\ ${ }^{4}$ Department of Astronomy \& Oskar Klein Centre, Stockholm University, Albanova, SE-10691 Stockholm, Sweden \\ ${ }^{5}$ Centre for Astrophysics \& Supercomputing, Swinburne University of Technology, Hawthorn, Victoria 3122, Australia \\ Received 2012 June 20; accepted 2012 July 31; published 2012 August 14
}

\begin{abstract}
The intergalactic medium was reionized before redshift $z \sim 6$, most likely by starlight which escaped from early galaxies. The very first stars formed when hydrogen molecules $\left(\mathrm{H}_{2}\right)$ cooled gas inside the smallest galaxies, minihalos (MHs) of mass between $10^{5}$ and $10^{8} M_{\odot}$. Although the very first stars began forming inside these MHs before redshift $z \sim 40$, their contribution has, to date, been ignored in large-scale simulations of this cosmic reionization. Here we report results from the first reionization simulations to include these first stars and the radiative feedback that limited their formation, in a volume large enough to follow the crucial spatial variations that influenced the process and its observability. We show that, while MH stars stopped far short of fully ionizing the universe, reionization began much earlier with $\mathrm{MH}$ sources than without, and was greatly extended, which boosts the intergalactic electron-scattering optical depth and the large-angle polarization fluctuations of the cosmic microwave background significantly. This boost should be readily detectable by Planck, although within current Wilkinson Microwave Anisotropy Probe uncertainties. If reionization ended as late as $z_{\mathrm{ov}} \lesssim 7$, as suggested by other observations, Planck will thereby see the signature of the first stars at high redshift, currently undetectable by other probes.
\end{abstract}

Key words: cosmology: theory - galaxies: high-redshift - radiative transfer

Online-only material: color figures

\section{INTRODUCTION}

The theory of reionization has not yet advanced to the point of establishing unambiguously its timing and the relative contributions to it from galaxies of different masses. In a cold dark matter ("CDM") universe, these early galactic sources can be categorized by their host halo mass into minihalos ("MHs") and "atomic-cooling" halos ("ACHs"). MHs have masses $M \sim 10^{5}-10^{8} M_{\odot}$ and virial temperatures $T_{\text {vir }} \sim 10^{4} \mathrm{~K}$, and thus molecular hydrogen $\left(\mathrm{H}_{2}\right)$ was necessary to cool the gas below this virial temperature to begin star formation. $\mathrm{ACHs}$ have $M \gtrsim 10^{8} M_{\odot}$ and $T_{\text {vir }} \gtrsim 10^{4} \mathrm{~K}$ for which $\mathrm{H}$-atom radiative line cooling alone was sufficient to support star formation. The ACHs can be split further into low-mass atomic-cooling halos ("LMACHs"; $M \sim 10^{8}-10^{9} M_{\odot}$ ), for which the gas pressure of the photoionization-heated intergalactic medium ("IGM") in an ionized patch prevented the halo from capturing the gas it needed to form stars, and high-mass atomic-cooling halos ("HMACHs"; $M \gtrsim 10^{9} M_{\odot}$ ), for which gravity was strong enough to overcome this "Jeans-mass filter" and form stars even in the ionized patches.

Once starlight escaped from galactic halos into the IGM to reionize it, the ionized patches ("H II regions") of the IGM became places in which star formation was suppressed in both MHs and LMACHs. At the same time, UV starlight at energies in the range of 11.2-13.6 eV also escaped from the halos, capable of destroying the $\mathrm{H}_{2}$ molecules inside $\mathrm{MHs}$ through Lyman-Werner band ("LW") dissociation, even in the neutral zones of the IGM. This dissociation eventually prevented further star formation in some of the MHs where the background intensity was high enough. Early estimates, in fact, suggested that this would have made the MH contribution to reionization small (Haiman et al. 2000), and, until now, largescale simulations of reionization have neglected them altogether.
In this Letter, we report the first radiative transfer (RT) simulations of reionization to include all three of the mass categories of reionization source halos, along with their radiative suppression, in a simulation volume large enough to capture both the global mean ionization history and the observable consequences of its evolving patchiness in a statistically meaningful way. ${ }^{6} \mathrm{We}$ overcame the limitation of previous large-volume simulations by applying a newly developed sub-grid treatment to include MH sources (Section 2) and calculating the transfer of LW-band radiation self-consistently with the source population using the scheme of Ahn et al. (2009).

\section{METHODS}

We performed a cosmological $N$-body simulation of structure formation with $3072^{3}$ particles in a $114 h^{-1} \mathrm{Mpc}$ simulation box, using the WMAP5 background cosmology (Dunkley et al. 2009). For this we used the code Cube $\mathrm{P}^{3} \mathrm{M}$ (Merz et al. 2005; Illiev et al. 2008; J. Harnois-Deraps et al. 2012, in preparation) in which the gravity is computed by a particle-particle-particlemesh $\left(\mathrm{P}^{3} \mathrm{M}\right)$ scheme. The simulation was started at redshift $z=300$ and run to $z=6 . N$-body data were recorded at 86 equally spaced times (every $11.53 \mathrm{Myr}$ ) from $z=50$ to $z=6$. Each data time slice was then used to create matter density fields by smoothing the particle data adaptively onto a uniform mesh - or an "RT grid" - of $256^{3}$ cells. All cosmological halos with $M \geqslant 10^{8} M_{\odot}$ (corresponding to 20 particles or more), and thus both LMACHs and HMACHs, were identified on the fly using a spherical overdensity halo finder with overdensity of $\Delta=178$ with respect to the mean.

\footnotetext{
6 First results of these simulations were briefly summarized in Ahn et al. (2010).
} 

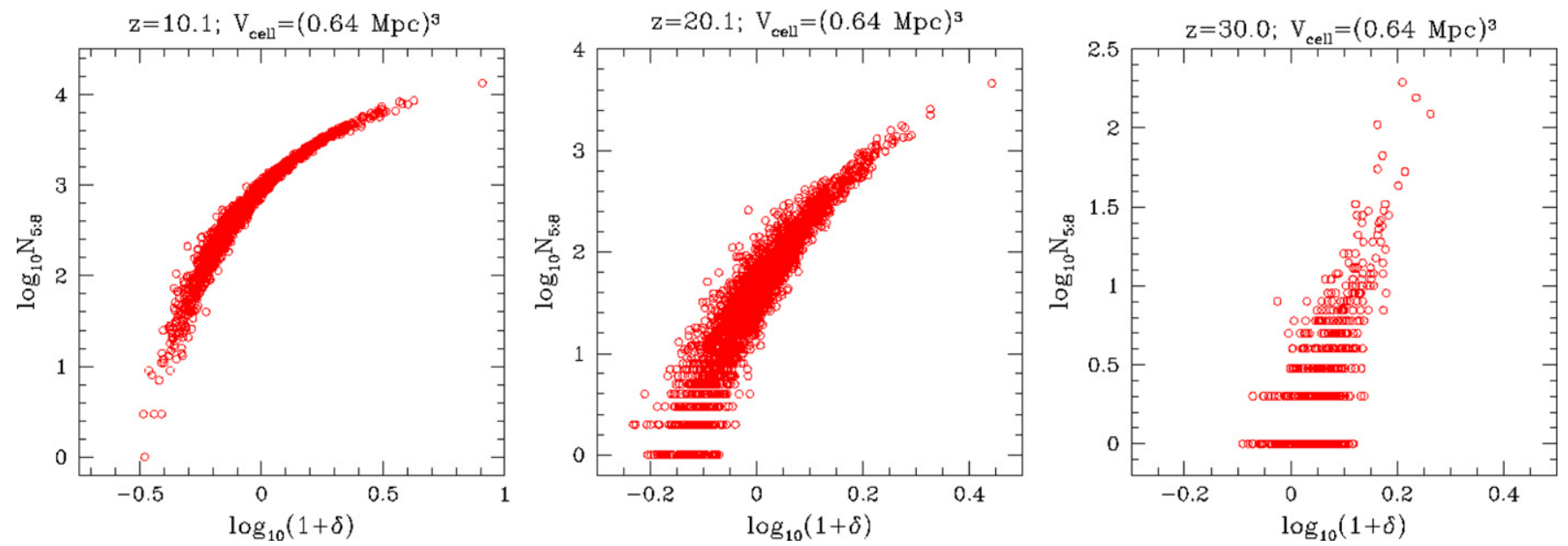

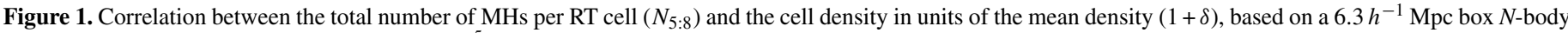

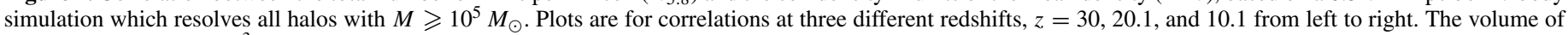
the RT cell is $(0.64 \mathrm{Mpc})^{3}$.

(A color version of this figure is available in the online journal.)

Because MHs are too small to be resolved in our simulation box, our RT grid was populated with MHs through a newly developed sub-grid model, as follows. We started with a separate, high-resolution $\mathrm{N}$-body simulation of structure formation in a box with $\left(6.3 h^{-1} \mathrm{Mpc}\right)^{3}$ volume and $1728^{3}$ particles, which resolved all MHs with $M \geqslant 10^{5} M_{\odot}$ with 20 particles or more. We then partitioned the box into a uniform grid of $14^{3}$ cells, such that each cell is the same size as one of the RT grid cells in our main, $114 h^{-1} \mathrm{Mpc}$ simulation box, and calculated cell density and the total number of MHs per cell. A strong and tight correlation between the number of MHs located in a cell and its density is observed (Figure 1). The best fit to this correlation at each redshift was then used as the total number of MHs in each grid cell of $114 h^{-1} \mathrm{Mpc}$ box.

Based on these structure formation results for the IGM density field and the source dark matter halos, we then calculated the RT of $\mathrm{H}$-ionizing and $\mathrm{H}_{2}$-dissociating photons (see Table 1 for the RT simulation parameters). The stars inside ACHs are assumed to produce $g_{\gamma}$ ionizing photons per baryon every $10 \mathrm{Myr}$, where $g_{\gamma} \equiv f_{\gamma} /\left(t_{\star} / 10 \mathrm{Myr}\right)$, and where $f_{\gamma} \equiv f_{\mathrm{e}} f_{\star} N_{\mathrm{i}}, f_{\mathrm{e}}$ is the escape fraction of ionizing photons, $f_{\star}$ is the star formation efficiency, and $N_{\mathrm{i}}$ is the number of ionizing photons per stellar baryon produced over the star's lifetime $t_{\star}$-we use $t_{\star}=11.53 \mathrm{Myr}$ for both HMACHs and LMACHs, and $t_{\star}=1.92 \mathrm{Myr}$ for MHs. We assign one Pop III star per MH, motivated by numerical simulations of first star formation inside $\mathrm{MHs}$, which find that typically one Pop III star with a mass between 100 and $1000 M_{\odot}$ forms per $\mathrm{MH}$ in the absence of strong soft UV radiative feedback (Bromm et al. 2002; Abel et al. 2002; Yoshida et al. 2006). At each cell, only those MHs which are newly collapsed every 1.92 Myr are assumed to host Pop III stars to roughly approximate the disruptive radiative and mechanical feedback by the first star and its by-products (such as a supernova) on the halo gas (for this, we create "morphed" density fields every 1.92 Myr by linearly interpolating the $N$-body density fields in time which are separated by a time interval of $11.53 \mathrm{Myr}$ and finite difference corresponding $\mathrm{MH}$ populations on each cell). Star formation in MHs is further suppressed when the local LW background - calculated at each time step in three dimensions using the scheme by Ahn et al. (2009), but now considering both ACHs and MHs and also improved in speed using the fast
Fourier transform (FFT) scheme-reaches a certain threshold $J_{\mathrm{LW}}$. th. At present, the precise value of this threshold is not well determined, but the typical values found by high-resolution simulations of MH star formation are $J_{\mathrm{LW}, \text { th }}=[0.01-0.1] \times$ $10^{-21} \mathrm{erg} \mathrm{s}^{-1} \mathrm{~cm}^{-2} \mathrm{sr}^{-1}$ (Machacek et al. 2001; Yoshida et al. 2003; O'Shea \& Norman 2008). We adopted a constant value chosen from this range for each simulation. Even though stars may still form by $\mathrm{H}_{2}$ cooling, when $J_{\mathrm{LW}}>J_{\mathrm{LW}}$, th , in MHs in the mass range $M \simeq 2 \times 10^{6}-10^{8} M_{\odot}$ and $M \simeq 10^{7}-10^{8} M_{\odot}$ at $J_{\mathrm{LW}, \text { th }} \simeq 0.01 \times 10^{-21} \mathrm{erg} \mathrm{s}^{-1} \mathrm{~cm}^{-2} \mathrm{sr}^{-1}$ and $J_{\mathrm{LW} \text {, th }} \simeq 0.1 \times$ $10^{-21} \mathrm{erg} \mathrm{s}^{-1} \mathrm{~cm}^{-2} \mathrm{sr}^{-1}$, respectively (e.g., O'Shea \& Norman 2008), we neglect their contribution because they constitute only a small fraction of the whole $\mathrm{MH}$ population.

The simulations with ACHs only (and without LW RT) are described in Iliev et al. (2012). Simulation parameters are given in Table 1.

\section{ROLE OF THE FIRST STARS DURING COSMIC REIONIZATION}

We demonstrate the effects of the first stars by direct comparison of the results from two simulations: a fiducial case which includes all ionizing sources down to the first stars hosted by MHs (case L2M1J1) and a corresponding reference case which includes the larger, atomically cooling halos with exactly the same properties, but no MH sources (case L2, previously presented in Iliev et al. 2012). Our results show that the early reionization history is completely dominated by the first stars, while the late (redshift $z \lesssim 10$ ) history is driven by the stars inside HMACHs (Figures 2 (a) and (b), top panel). The very first stars start to form inside MHs at redshift $z \simeq 40$ and dominate the reionization process until $z \simeq 10$ but through self-regulation which slows their contribution to reionization ${ }^{7}$ (Figure 2(b)). Although the abundance of ACHs rises exponentially, they remain relatively rare, and thus sub-dominant, until $z \simeq 10$. After redshift $z \simeq 8$, though, the two reionization histories become

\footnotetext{
7 The oscillation of $J_{\mathrm{LW}}$ around the plateau at $J_{\mathrm{LW}} / J_{\mathrm{LW}}$, th $\sim 1$ observed in Figures 2(b) and 3 is a numerical artifact which occurs because LW suppression locks the $\mathrm{MH}$ star formation rate onto the level that keeps $J_{\mathrm{LW}}=J_{\mathrm{LW}}$, th , and the simulation time step (1.92 Myr) is comparable to the $\mathrm{MH}$ formation timescale.
} 
Table 1
Reionization Simulation Source Halo Properties and Global History Results

\begin{tabular}{|c|c|c|c|c|c|c|c|c|}
\hline Case & $g_{\gamma, \mathrm{H}}\left(f_{\gamma, \mathrm{H}}\right)$ & $g_{\gamma, \mathrm{L}}\left(f_{\gamma, \mathrm{L}}\right)$ & $g_{\gamma, \mathrm{MH}}\left(f_{\gamma, \mathrm{MH}}\right)$ & $M_{\text {III, },}$ & $J_{\mathrm{LW}, \text { th }}$ & $z_{\mathrm{ov}}$ & $\tau_{\mathrm{es}}$ & $m_{1}, m_{2}, \ldots, m_{7}$ \\
\hline$g 8.7 \_130 S$ (L1) & $8.7(10)$ & $130(150)$ & $\ldots$ & $\ldots$ & $\ldots$ & 8.40 & 0.0841 & $-0.298,-0.0267,0.289,0.115,0.0975,0.0918,-0.0548$ \\
\hline$g 8.7 \_130 S_{-} M 300 \_J 0.05$ (L1M1J2) & $8.7(10)$ & $130(150)$ & $5063(1013)$ & 300 & 0.05 & 8.41 & 0.0934 & $-0.283,-0.0222,0.268,0.121,0.0828,0.0897,-0.0565$ \\
\hline$g 8.7 \_130 S_{-} M 100 \_J 0.05$ (L1M2J2) & $8.7(10)$ & $130(150)$ & $1687.7(337.7)$ & 100 & 0.05 & 8.41 & 0.0910 & $-0.288,-0.0234,0.274,0.120,0.0868,0.0908,-0.0558$ \\
\hline g8.7_130S_M300_J0.01 (L1M1J3) & $8.7(10)$ & $130(150)$ & $5063(1013)$ & 300 & 0.01 & 8.41 & 0.0874 & $-0.293,-0.0236,0.283,0.118,0.0952,0.0919,-0.0541$ \\
\hline$g 8.7 \_130 S_{-} M 100 \_J 0.01(\mathrm{~L} 1 \mathrm{M} 2 \mathrm{~J} 3)$ & $8.7(10)$ & $130(150)$ & $1687.7(337.7)$ & 100 & 0.01 & 8.41 & 0.0861 & $-0.295,-0.0247,0.285,0.117,0.0962,0.0918,-0.0545$ \\
\hline$g 1.7 \_8.7 \mathrm{~S}(\mathrm{~L} 2)$ & $1.7(2)$ & $8.7(10)$ & $\ldots$ & $\ldots$ & $\ldots$ & 6.76 & 0.0603 & $-0.298,0.00402,0.372,0.191,0.0446,0.0229,-0.0416$ \\
\hline$g 1.7 \_8.7 \mathrm{~S} \_M 300 \_J 0.1$ (L2M1J1) & $1.7(2)$ & $8.7(10)$ & $5063(1013)$ & 300 & 0.1 & 6.80 & 0.0861 & $-0.276,-0.00969,0.302,0.158,0.0260,0.00619,-0.0349$ \\
\hline$g 1.7 \_8.7 \mathrm{~S} \_M 300 \_J 0.05$ (L2M1J2) & $1.7(2)$ & $8.7(10)$ & $5063(1013)$ & 300 & 0.05 & 6.79 & 0.0788 & $-0.281,-0.00479,0.323,0.170,0.0285,0.00893,-0.0377$ \\
\hline
\end{tabular}

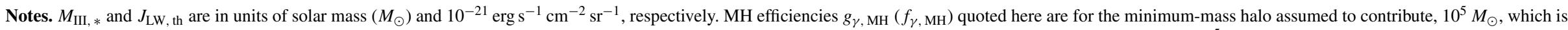
roughly comparable to the average value for the MHs integrated over the halo mass function. The efficiency of the MH of mass $M$ is obtained simply by multiplying $\left(10^{5} M_{\odot} / M\right)$ to the quoted $g_{\gamma}, \mathrm{MH}\left(f_{\gamma, \mathrm{MH}}\right)$. 

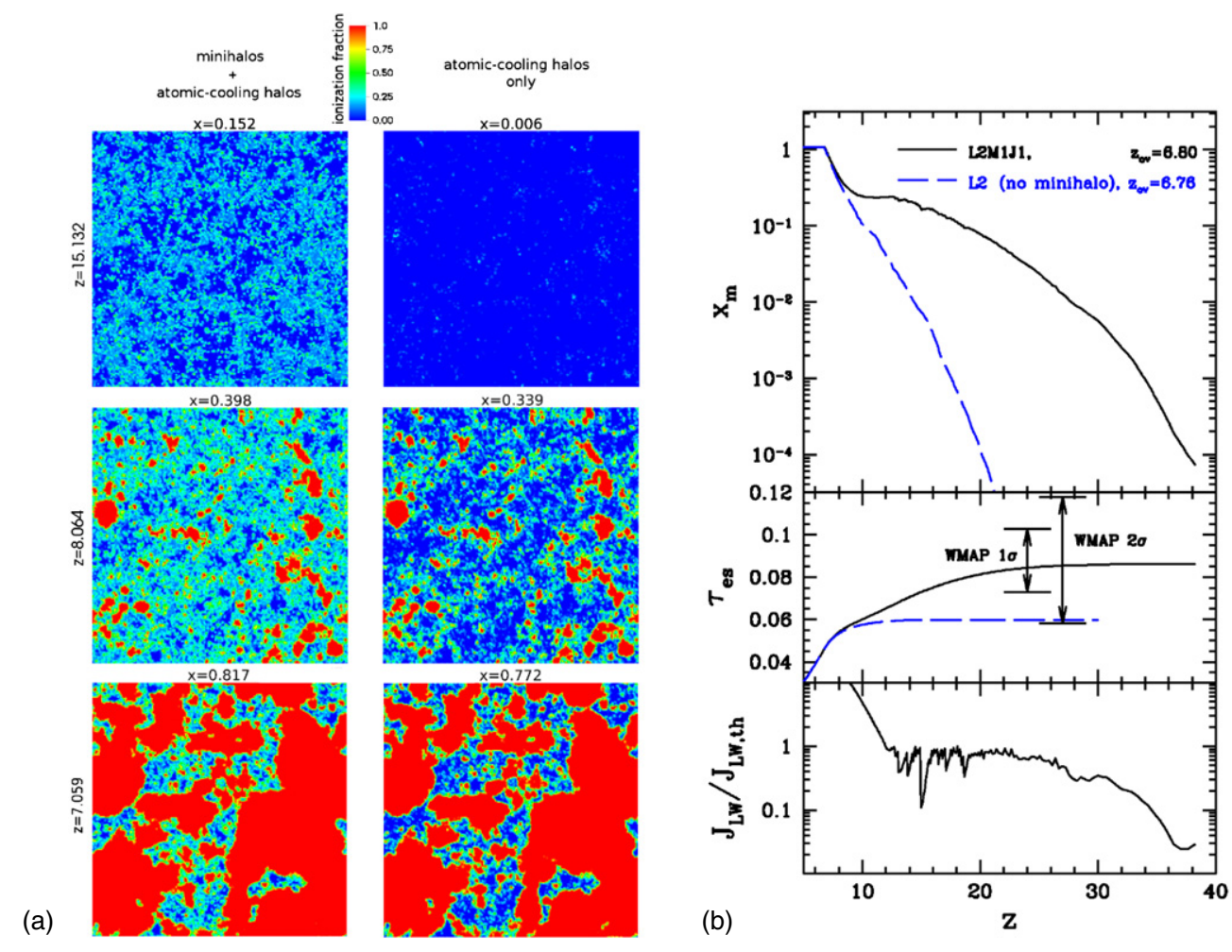

Figure 2. (a) Maps of evolving hydrogen-ionized fractions at different redshifts (rows), for our fiducial model with MH sources included, L2M1J1 (first column), vs. the corresponding reference model with only atomically cooling halos, case L2 (second column). The slices are $0.45 h^{-1} \mathrm{Mpc}$ thick. Color represents linearly scaled ionized fraction from 0 (blue) to 1 (red). (b) Top: globally averaged history of the mass-weighted ionized fraction for models L2M1J1 (black, solid) and L2 (blue, dashed). Middle: $\tau_{\mathrm{es}}$ integrated from $z=0$ to redshift $z$ for $\mathrm{L} 2$ and L2M1J1. Bottom: evolution of the mean $J_{\mathrm{LW}}$ in units of $J_{\mathrm{LW}}$, th for case L2M1J1.

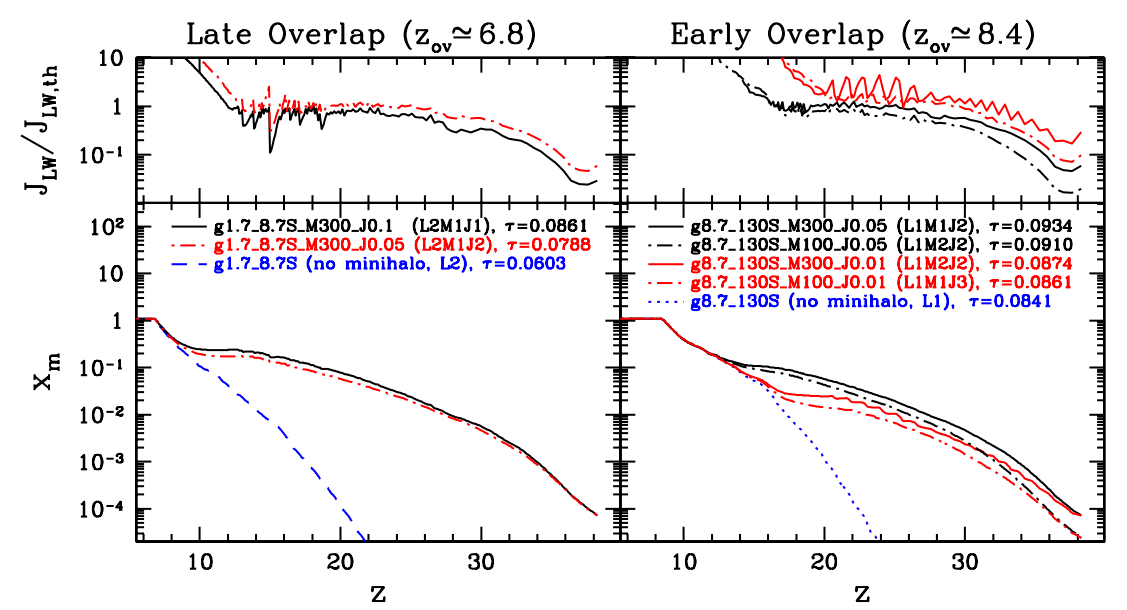

Figure 3. Model dependency of the history of cosmic reionization. In addition to cases L1 and L2, which do not account for MHs, we show predictions for MH-included cases by parameterizing the star formation inside MHs through $M_{*, \text { III }}$ (mass of the Pop III star) and $J_{\mathrm{LW}}$, th (threshold Lyman-Werner intensity). Left: late-overlap models. Right: early-overlap models.

largely indistinguishable because the same HMACHs then dominate reionization and push $J_{\mathrm{LW}}$ above $J_{\mathrm{LW}}$, th (at $z \simeq 12$ ), halting $\mathrm{MH}$ star formation altogether, long before the MHs can complete reionization on their own.

Nonetheless, the MH sources (the first stars) can have quite a dramatic effect on the electron-scattering optical depth $\tau_{\mathrm{es}}$. While intergalactic $\mathrm{H}$ II regions fully overlap (at redshift $z_{\mathrm{ov}}$, here defined as when the mass-weighted mean ionized fraction in the IGM, $x_{m}$, first surpassed 99\%) at almost identical redshifts $z_{\mathrm{ov}} \simeq 6.8$ with (L2M1J1) or without (L2) MHs, the early rise of $x_{m}$ with MH sources boosts the optical depth by as much as $47 \%$ relative to that without MH sources: $\tau_{\mathrm{es}}=0.0861$ for L2M1J1, while $\tau_{\mathrm{es}}=0.0603$ for L2. This satisfies the current observational constraints on reionization: (1) reionization ended no earlier than redshift $z=7$ (Fan \& et al. 2006; Ota et al. 2010; Mortlock et al. 2011; Bolton et al. 2011; Pentericci et al. 2011) and (2) $\tau_{\mathrm{es}}=0.088 \pm 0.015$ at $68 \%$ confidence level (Larson et al. 2011). Predicted values of $\tau_{\mathrm{es}}$ and $z_{\mathrm{ov}}$ are model dependent, and thus we tested the robustness of our conclusions by varying the physical parameters of MHs and ACHs (Figure 3).

The first stars, born inside MHs, imprint a distinctive pattern on the global reionization history. For example, in case L2M1J1, 

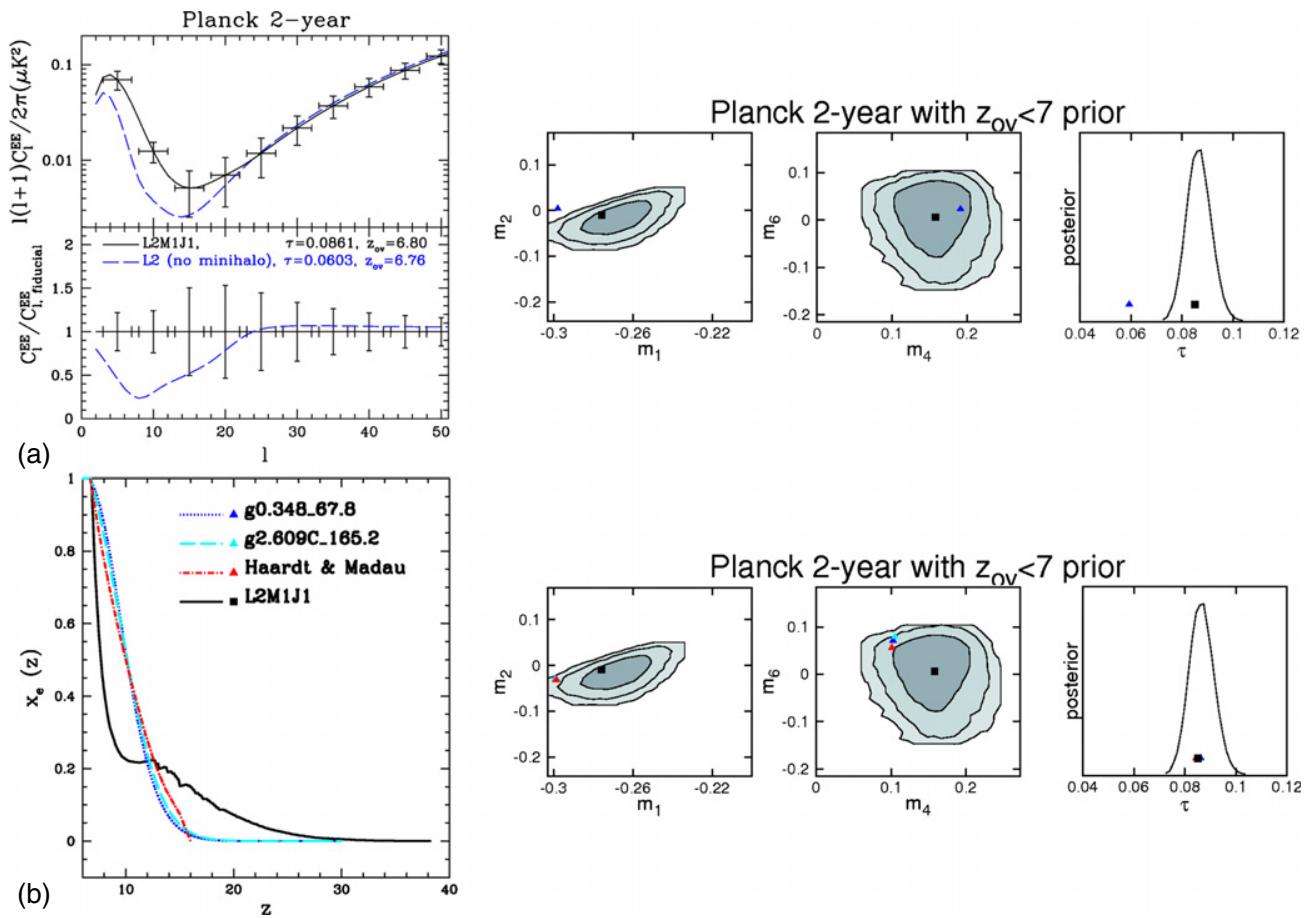

Figure 4. (a) Detecting the first stars. Left: forecasts of $C_{l}^{\mathrm{EE}}$ of cases L2M1J1 (with MHs) and L2 (no MHs) for Planck. The error bars are estimated Planck two year $1 \sigma$ sensitivity including cosmic variance (top panel; The Planck Collaboration 2006). Right: model-selection power of Planck. Contours represent $1 \sigma$ (68\%), $2 \sigma$ (95\%), and $3 \sigma(99.7 \%)$ confidence levels from inside out, on marginalized posterior distributions of selected parameters $\left(m_{\mu}\right.$ 's and $\left.\tau_{\text {es }}\right)$ using mock data based upon Model L2M1J1 (black square). Case L2 (blue triangle) can be ruled out only from the measurement of $\tau_{\mathrm{es}}$ by Planck. The prior condition of $z_{\mathrm{ov}} \leqslant 7$ is applied, which rules out early reionization $\left(z_{\mathrm{ov}} \gtrsim 8\right)$ models. (b) Breaking the degeneracy in $z_{\mathrm{ov}}$ and $\tau_{\mathrm{es}}$. Left: ionization histories of various models, but with identical $z_{\mathrm{ov}}(\simeq 6.8)$ and $\tau_{\mathrm{es}}(\simeq 0.085$ ). The model with MH sources (case L2M1J1, black line) stands out from almost identical, no-MH models. g0.348_67.8 (no clumping; blue, dotted) and $g$ 2.609C_165.2 (with $z$-dependent clumping; cyan, dashed) are semi-analytic models obtained from Equation (A1) of Iliev et al. (2007) with $n=0.1$, and Haardt \& Madau (red, dot-dashed) is from Haardt \& Madau (2012). Right: hypothesis-testing power of Planck on MH-included (black square) vs. no-MH models (triangles). Contours have the same meaning as those in (A). No-MH models are clustered and well separated from case L2M1J1 at $\gtrsim 2 \sigma$ confidence level.

(A color version of this figure is available in the online journal.)

when the LW plateau ended, reionization briefly stalled, since MHs no longer formed the stars which replenished the ionizing background and only ACH sources remained, thereafter; the $\mathrm{ACH}$ contribution took a bit more time to climb enough to move reionization forward again. This explains the brief " $x_{m}$-plateau" from $z \sim 12$ to $z \sim 10$ in Figure 2(b) for case L2M1J1, while in case L2, $x_{m}$ grows continuously without showing such a plateau (Figure 2(b)). This feature is generic (see Figures 3 and 4(b) for different sets of parameters we explored). Reionization histories without $\mathrm{MH}$ sources, modeled either by large-scale RT simulations (Ciardi et al. 2003; Iliev et al. 2006, 2007; Trac \& Cen 2007; McQuinn et al. 2007; Zahn et al. 2007; Iliev et al. 2012) or semi-analytical calculations (Haiman et al. 2000; Zahn et al. 2007), are all similar in that respect.

Reionization histories with $\mathrm{MH}$ sources calculated here, however, find an ionization plateau phase. Previous studies that considered MH stars and their impact were not able to settle the issue of their global effect on reionization. This is either because they simulated volumes much too small to represent a fair portion of the universe (Ricotti et al. 2002; Sokasian et al. 2004; Yoshida et al. 2006; Ricotti et al. 2008; Johnson et al. 2012) or else treated reionization by a semi-analytical, 1-zone, homogeneous approximation (either with LW suppression included (Haiman et al. 2000; Furlanetto \& Loeb 2005) or without (Shapiro et al. 1994; Wyithe \& Loeb 2003; Haiman \& Bryan 2006; Wyithe \& Cen 2007)), which cannot capture its innate spatially inhomogeneous nature, or made a semi-analytical approximation that accounted statistically for spatial inhomogeneity but without LW suppression (Kramer et al. 2006).
We find that the global reionization history at $z \lesssim 20$ depends on $J_{\mathrm{LW}}$, th more strongly than $M_{\star, \text { III. }}$. This is due to the very nature of self-regulation of the first star formation. The larger the $J_{\mathrm{LW}}$, th is, the weaker the suppression of star formation becomes, thereby temporarily hastening the progress of reionization. If $M_{\star, \text { III }}$ is smaller (Turk et al. 2009; Stacy et al. 2010; Greif et al. 2011a; Stacy et al. 2012) than those simulated here, those stars produce less ionizing and LW radiation and the resulting suppression is weaker, which partly compensates for the lower emission per star. Similar type of compensation would occur also when the number of MHs with a potential to form the first stars is smaller than our estimate due to the relative offset of baryonic gas from some of the MHs (Tseliakhovich \& Hirata 2010; Greif et al. 2011b).

\section{PROBING THE FIRST STARS WITH PLANCK}

We thus conclude that the first stars hosted by MHs likely made an important contribution to reionization. But how can we probe them observationally? While significant, the effects of the first stars are largely confined to the early stages of reionization, at redshifts $z>10$, which puts them beyond the reach of most current instruments. Recent observations by the South Pole Telescope (SPT) have been used to place an upper limit on the kinetic Sunyaev-Zel'dovich (kSZ) effect from the epoch of reionization (Reichardt et al. 2012). While it has been suggested that this restricts the duration of reionization (Zahn et al. 2011), we will show elsewhere (H. Park et al. 2012, in preparation) that the kSZ signal from our fiducial case, L2M1J1, is well 
below the observed upper bound. The combined effect of the first stars will be reflected in the cosmic microwave background (CMB) polarization anisotropies at large scales. The current best constraints on $\tau_{\mathrm{es}}$ by the Wilkinson Microwave Anisotropy Probe satellite are still relatively weak, and thus models with low $\tau_{\mathrm{es}}$ values like L2 are still acceptable at the $2 \sigma(95 \%)$ confidence level (Figure 2(b), middle panel). However, through the far more precise measurement of the CMB polarization by the Planck mission we should be able to discern the influence of the first stars on reionization.

As we discussed above, current observational constraints suggest that reionization was not complete before $z \sim 7$. Imposing this condition as a prior on the allowed reionization histories $x_{m}(z)$, we predict that the Planck mission will clearly detect the era of first stars (Figure 4). In Figure 4, we show a statistical measure of the Planck sensitivity in detecting the signature of the first stars through the principal component analysis by $\mathrm{Hu}$ \& Holder (2003) and Mortonson \& Hu (2008, who modified COSMOMC (Lewis \& Bridle 2002) to allow generic reionization models). ${ }^{8}$ Reionization principal components $\left\{S_{\mu}(z)\right\}$ are eigenvectors of the relevant Fisher matrix (evaluated with an arbitrarily chosen fiducial history $\left.x_{e, \text { fid }}(z)\right)$,

$$
F_{i j} \equiv \sum_{l=2}^{l_{\max }}\left(l+\frac{1}{2}\right) \frac{\partial^{2} C_{l}^{\mathrm{EE}}}{\partial x_{e}\left(z_{i}\right) \partial x_{e}\left(z_{j}\right)},
$$

which can be used to describe any generic ionization history $x_{e}(z)$ with just a small number of modes, such that

$$
x_{e}(z)=x_{e, \mathrm{fid}}(z)+\sum_{\mu=1}^{N_{\max }} m_{\mu} S_{\mu}(z) .
$$

The mode amplitude $m_{\mu}$, for a given history $x_{e}(z)$, becomes

$$
m_{\mu}=\frac{\int_{z_{\min }}^{z_{\max }} d z S_{\mu}(z)\left[x_{e}(z)-x_{e, \mathrm{fid}}(z)\right]}{z_{\max }-z_{\min }} .
$$

Based on the Planck data after its full two years of planned operation, the narrow posterior distribution of allowed $\tau_{\mathrm{es}}$ values will allow us to distinguish reionization models like L2 and L2M1J1 unambiguously, and thereby strongly constrain the available reionization models. A high measured value of $\tau_{\mathrm{es}}>0.085$ will be a clear (if indirect) signature of the first stars.

Finally, we note that the presence of $\mathrm{MH}$ sources introduces the $x_{m}$ plateau noted above, which in turn imprints characteristic features on $C_{l}^{\mathrm{EE}}$. Hence, Planck might be able to distinguish (albeit at lower statistical significance, of $\gtrsim 2 \sigma$ or $\gtrsim 95 \%$ ) reionization models with and without first stars even if they have

\footnotetext{
8 While we use the scheme by Mortonson \& Hu (2008), we implement the following ingredients to optimize the analysis for our purpose. First, to apply the late-reionization prior, $z_{\mathrm{ov}} \leqslant 7$, we created seven sets of principal components based on $x_{e}$, fid $(z)=(40-z) /(40-6.5)$ (see Equation (2) for the definition of $x_{e}$, fid $(z)$ ), which make $x_{e}(z)$ behave well around $z \simeq z_{\mathrm{ov}}$. We then use this late-reionization prior to reject any sample reionization history with $z_{\mathrm{ov}}>7$ when forming the Monte Carlo Markov Chain of varying reionization models. Second, we improve the physicality condition, or $0 \leqslant x_{e}(z) \leqslant 1$ at any $z$, which was somewhat poorly applied in Mortonson \& Hu (2008). Whenever a set of $m_{\mu}$ parameters (Equation (3)) are sampled, we calculate the corresponding $x_{e}(z)$, and when either $\min \left(x_{e}(z)\right)>0.04$ or $\max \left(x_{e}(z)\right)<1.04$ is violated, we reject that sample. This small, $4 \%$ non-physicality in $x_{e}$ is still necessary because of the oscillatory nature of $x_{e}(z)$ caused by the limited number of principal components, but has only modest effects on the CMB E-mode polarization power spectrum $C_{l}^{\mathrm{EE}}$.
}

very similar values of $\tau_{\mathrm{es}}$ and $z_{\mathrm{ov}}$ (Figure 4(b)). Full reionization simulations like ours find it hard to satisfy both of these observational constraints without including a significant contribution from the first stars, but some semi-analytical models (Haiman \& Bryan 2006; Haardt \& Madau 2012; g0.348_67.8 and $g 2.609 C_{-} 165.2$ in Figure 4(b) which are of unnaturally large gaps in relative efficiencies of LMACH and HMACH) do find such scenarios. However, all such models lack the plateau feature in $x_{m}(z)$, regardless of the details of the assumed physics, and reside in a narrow window of the $m_{\mu}$-parameter space adjacent to that occupied by our no-MH cases, as demonstrated in Figure 4(b).

In summary, Planck is capable of distinguishing with high confidence between definitive classes of reionization scenarios allowed by the current constraints, and thereby significantly restricting the available parameter space. Planck will either probe the signature of the first stars or show that the first stars had a negligible impact on reionization. Once these first results confirm the role of the first stars, simulations of the type presented here can be used to study other observable quantities and thus deepen our understanding of the early universe.

K.A. was supported in part by NRF grant funded by the Korean government MEST (Nos. 2009-0068141, 20090076868, 2012R1A1A1014646, and 2012M4A2026720). I.T.I. was supported by The Southeast Physics Network (SEPNet) and the Science and Technology Facilities Council grants ST/F002858/1 and ST/I000976/1. This study was supported in part by the Swedish Research Council grant 2009-4088; U.S. NSF grants AST-0708176 and AST-1009799; NASA grants NNX07AH09G, NNG04G177G, and NNX11AE09G; and Chandra grant SAO TM8-9009X. The authors acknowledge the TeraGrid and the Texas Advanced Computing Center (TACC) at The University of Texas at Austin (URL: http://www.tacc.utexas.edu), and the Swedish National Infrastructure for Computing (SNIC) resources at HPC2N (Umeå, Sweden) for providing HPC and visualization resources that have contributed to the results reported within this paper. We acknowledge A. Lewis, A. Liddle, and M. Mortonson for scientific and technical input on Planck forecasts (Lewis, Liddle) and modifying COSMOMC for reionization principal components (Mortonson).

\section{REFERENCES}

Abel, T., Bryan, G. L., \& Norman, M. L. 2002, Science, 295, 93

Ahn, K., Shapiro, P. R., Iliev, I. T., Mellema, G., \& Pen, U. 2009, ApJ, 695, 1430

Ahn, K., Shapiro, P. R., Iliev, I. T., et al. 2010, in AIP Conf. Proc. 1294, The First Stars and Galaxies: Challenges for the Next Decade, ed. D. J. Whalen, V. Bromm, \& N. Yoshida (Melville, NY: AIP), 250

Bolton, J. S., Haehnelt, M. G., Warren, S. J., et al. 2011, MNRAS, 416, L70

Bromm, V., Coppi, P. S., \& Larson, R. B. 2002, ApJ, 564, 23

Ciardi, B., Stoehr, F., \& White, S. D. M. 2003, MNRAS, 343, 1101

Dunkley, J., Komatsu, E., Nolta, M. R., et al. 2009, ApJS, 180, 306

Fan, X., Strauss, M. A., Becker, R. H., et al. 2006, AJ, 132, 117

Furlanetto, S. R., \& Loeb, A. 2005, ApJ, 634, 1

Greif, T. H., Springel, V., White, S. D. M., et al. 2011a, ApJ, 737, 75

Greif, T. H., White, S. D. M., Klessen, R. S., \& Springel, V. 2011b, ApJ, 736, 147

Haardt, F., \& Madau, P. 2012, ApJ, 746, 125

Haiman, Z., Abel, T., \& Rees, M. J. 2000, ApJ, 534, 11

Haiman, Z., \& Bryan, G. L. 2006, ApJ, 650, 7

Hu, W., \& Holder, G. P. 2003, Phys. Rev. D, 68, 023001

Iliev, I. T., Mellema, G., Pen, U.-L., et al. 2006, MNRAS, 369, 1625

Iliev, I. T., Mellema, G., Shapiro, P. R., \& Pen, U. 2007, MNRAS, 376, 534

Iliev, I. T., Mellema, G., Shapiro, P. R., et al. 2012, MNRAS, 423, 2222 
Iliev, I. T., Shapiro, P. R., Mellema, G., Merz, H., \& Pen, U.-L. 2008, arXiv:0806.2887

Johnson, J. L., Dalla Vecchia, C., \& Khochfar, S. 2012, arXiv:1206.5824

Kramer, R. H., Haiman, Z., \& Oh, S. P. 2006, ApJ, 649, 570

Larson, D., Dunkley, J., Hinshaw, G., et al. 2011, ApJS, 192, 16

Lewis, A., \& Bridle, S. 2002, Phys. Rev. D, 66, 103511

Machacek, M. E., Bryan, G. L., \& Abel, T. 2001, ApJ, 548, 509

McQuinn, M., Lidz, A., Zahn, O., et al. 2007, MNRAS, 377, 1043

Merz, H., Pen, U.-L., \& Trac, H. 2005, New Astron., 10, 393

Mortlock, D. J., Warren, S. J., Venemans, B. P., et al. 2011, Nature, 474, 616

Mortonson, M. J., \& Hu, W. 2008, ApJ, 672, 737

O'Shea, B. W., \& Norman, M. L. 2008, ApJ, 673, 14

Ota, K., Iye, M., Kashikawa, N., et al. 2010, ApJ, 722, 803

Pentericci, L., Fontana, A., Vanzella, E., et al. 2011, ApJ, 743, 132

Reichardt, C. L., Shaw, L., Zahn, O., et al. 2012, ApJ, 755, 70

Ricotti, M., Gnedin, N. Y., \& Shull, J. M. 2002, ApJ, 575, 33
Ricotti, M., Gnedin, N. Y., \& Shull, J. M. 2008, ApJ, 685, 21 Shapiro, P. R., Giroux, M. L., \& Babul, A. 1994, ApJ, 427, 25 Sokasian, A., Yoshida, N., Abel, T., Hernquist, L., \& Springel, V. 2004, MNRAS, 350,47

Stacy, A., Greif, T. H., \& Bromm, V. 2010, MNRAS, 403, 45

Stacy, A., Greif, T. H., \& Bromm, V. 2012, MNRAS, 422, 290

The Planck Collaboration 2006, arXiv:astro-ph/0604069

Trac, H., \& Cen, R. 2007, ApJ, 671, 1

Tseliakhovich, D., \& Hirata, C. 2010, Phys. Rev. D, 82, 083520

Turk, M. J., Abel, T., \& O'Shea, B. 2009, Science, 325, 601

Wyithe, J. S. B., \& Cen, R. 2007, ApJ, 659, 890

Wyithe, J. S. B., \& Loeb, A. 2003, ApJ, 588, L69

Yoshida, N., Abel, T., Hernquist, L., \& Sugiyama, N. 2003, ApJ, 592, 645

Yoshida, N., Omukai, K., Hernquist, L., \& Abel, T. 2006, ApJ, 652, 6

Zahn, O., Lidz, A., McQuinn, M., et al. 2007, ApJ, 654, 12

Zahn, O., Reichardt, C. L., Shaw, L., et al. 2011, arXiv:1111.6386 